\title{
Comparative study on lipase production using solid state and submerged fermentation systems by several fungal strains and the predicted molecular characteristics
}

\begin{abstract}
Background: Lipases produced from microbial fermentation systems belong to the group of hydrolase enzymes and have tremendous commercial applications. The performance of solid state and submerged fermentation in producing lipase by several fungal strains was compared and the structure and functions of the lipases were predicted by molecular prediction studies. Methods: Lipase production was investigated using both solid state fermentation (SSF) and submerged liquid fermentation (SLF); with palm kernel cake as the fermentation substrate for SSF. Lipase activity, protease activity, total protein and biomass content were assayed. The predictive molecular characterization techniques were employed to determine possible structure and function of the lipases. Results: All fungal strains tested showed substantially higher lipase activity in SSF compared to SLF except for Trichoderma viride SDTC EDF 002. Aspergillus niger SDTC SRW-4 (44.43 U g-1; $176.36 \mathrm{mg}$ g-1) and Aspergillus niger DSMZ 2466 (42.05 U g-1; $176.28 \mathrm{mg}$ g-1) showed the highest lipase activities and total protein content. All lipases showed the presence of "lipase 3" domain with conserved dyad catalytic site of two residues (Ser-Asp) unlike several other esterase lipases with triad catalytic site (Ser-Asp/Glu-His). Predicted pI range of lipases was 4.22 to 8.28 with molecular weight ranging from $31.6 \mathrm{kDa}$ to $54.1 \mathrm{kDa}$. Conclusions: Production of lipase by Aspergillus niger SDTC SRW-4 and Aspergillus niger DSMZ 2466 using palm kernel cake as substrate was substantially higher in SSF as compared to SLF. Lipases produced by these fungus were predicted to have similar properties and functionality of triacylglycerol lipases (EC 3.1.1.3).
\end{abstract}

Keyword: Lipase; Aspergillus niger; Fermentation 